\title{
RELATIONSHIP BETWEEN DINUCLEAR SYSTEMS AND NUCLEI IN HIGHLY DEFORMED STATES
}

\author{
T.M.Shneidman ${ }^{1,2}$, G.G.Adamian ${ }^{1,2,3}$, N.V.Antonenko ${ }^{1,2}$, S.P.Ivanova ${ }^{1,2}$ and W.Scheid ${ }^{2}$ \\ ${ }^{1}$ Joint Institute for Nuclear Research, 141980 Dubna, Russia \\ ${ }^{2}$ Institut für Theoretische Physik der Justus-Liebig-Universität, D-35392 Giessen, Germany \\ ${ }^{3}$ Institute of Nuclear Physics, Tashkent 702132, Uzbekistan
}

(April 19, 2022)

\begin{abstract}
Potential energies, moments of inertia, quadrupole and octupole moments of dinuclear systems are compared with the corresponding quantities of strongly deformed nuclei. As dinuclear system we denote two touching nuclei (clusters). It is found that the hyperdeformed states of nuclei are close to those of nearly symmetric dinuclear systems, whereas the superdeformed states are considered as states of asymmetric dinuclear systems. The superdeformed and hyperdeformed states constructed from two touching clusters have large octupole deformations. The experimental measurement of octupole deformation of the highly deformed nuclei can answer whether these nuclei have cluster configurations as described by the dinuclear model.
\end{abstract}

PACS: 21.60.Ev,21.60.Gx

Key words: Dinuclear system; Cluster states in nuclei; Superdeformed and hyperdeformed states 


\section{INTRODUCTION}

One of the most important developments in nuclear structure physics was the prediction and observation of superdeformed (SD) [1] and hyperdeformed (HD) [2] nuclear shapes. The idea that nuclei could adopt highly deformed prolate shapes at low excitation energies was originated with the discovery of deformed isomers in the actinide region [3, 1 . Another group of superdeformed states with the ratio 3:2 of major to minor axis was discovered near the ground state in the $A \approx 76\left({ }^{72} \mathrm{Se},{ }^{74,76} \mathrm{Kr}\right)$ [5, 6] and $A \approx 100\left({ }^{98,100} \mathrm{Sr},{ }^{100} \mathrm{Zr}\right)$ [7],8 mass regions. In these nuclei the SD ground and excited states are strongly mixed with a spherical band which coexists at low spin [9]. While in the rare earth nuclei the highly deformed shapes are stabilized by collective rotation, the highly deformed nuclei exist even at zero spin. The states with large spins are populated in heavy ion fusion reactions. By the study of rotational bands one can determine the moments of inertia of highly deformed nuclei. Based on the experimental values of the moment of inertia, it was found that the SD and HD states are related to shapes with a ratio of axes of 2:1 and 3:1, respectively. Since the intensity of $\gamma$-transitions drastically decreases with decreasing angular momentum, the experimental determination of the excitation energy of the SD band is difficult.

In actinides the third potential minimum of the HD state is elucidated from a microstructure in the resonances found in the reactions $(n, f),(t, p f)$ and $(d, p f)$ [10. Another evidence supporting the existence of a third minimum is the observation of an asymmetric angular distribution of the light fission fragments of nuclei around ${ }^{232} \mathrm{Th}$ [11]. The transitions of odd multipolarity indicate a reflection-asymmetric shape of the nucleus ${ }^{232} \mathrm{Th}$.

Investigations of the SD and HD rotational bands in different mass regions were performed within semi-phenomenological cranked Woods-Saxon and Nilsson approaches [12,13]. Nuclear mean field theories, based on these approaches, well describe a rich variety of nuclear shapes all over the periodic table. These models allowed predictions of HD states which were recently observed experimentally [14]. The SD and HD rotational bands have been also investigated within relativistic mean field theories [15].

The nuclear shapes calculated within mean field theories are close to a rotational ellipsoid. It is known from the study of light nuclei $\left({ }^{8} \mathrm{Be},{ }^{32} \mathrm{~S}\right)$ that the SD shape of nuclei can be considered as a symmetric di-molecular shape rather than an ellipsoid. In light $\alpha$-particle nuclei the similarity between hyperdeformed and cluster-type states, i.e. quasi-molecular states, was already mentioned in [16,17]. Besides theoretical work, there are experimental evidences for the existence of the cluster-type configurations in fissioning nuclei [18]. The validity of the cluster approach for heavier nuclei has been investigated in [19 22]. An interesting observation in shell model calculations is that the nucleus in the third minimum corresponds to a dinuclear system (DNS) configuration [20]. However, in this model the clusters penetrate each other because the relative distance $R$ between the centers of the clusters is smaller than the sum of cluster radii $R_{1}+R_{2}$. As it was shown in [23, 24], the overlapping of nuclei is hindered by a repulsive potential at smaller relative distances $R$. Therefore, in the present paper we describe the SD and HD states by molecular-like dinuclear system configurations with a relative distance $R_{m} \approx R_{1}+R_{2}$, which corresponds to the minimum of the nucleus-nucleus potential [17]. In this paper we find a relationship between the DNS-type cluster configurations and highly deformed states of heavy nuclei. Consequences for assuming HD and SF states as cluster-type states are discussed. 


\section{MULTIPOLE MOMENTS OF DNS}

The mass $(k=m)$ and charge $(k=c)$ multipole moments of the DNS shape are calculated with the expression

$$
Q_{\lambda \mu}^{(k)}=\sqrt{\frac{16 \pi}{2 \lambda+1}} \int \rho^{(k)}(\mathbf{r}) r^{\lambda} Y_{\lambda \mu}(\Omega) d \tau
$$

For small overlaps of the nuclei in the DNS when $R \geq R_{1}+R_{2}$, where $R_{1}$ and $R_{2}$ are the radii of the nuclei and $R$ the distance between the centers of nuclei, the nuclear mass and charge densities $\rho^{(k)}$ in the DNS can be written as a sum of the densities in each nucleus (frozen density approximation):

$$
\rho^{(k)}(\mathbf{r})=\rho_{1}^{(k)}(\mathbf{r})+\rho_{2}^{(k)}(\mathbf{r}) .
$$

By using Eq.(2) and assuming axial symmetry of the nuclear shapes, the multipole moments of the DNS in the center of mass of system can be expanded in the following form:

$$
Q_{\lambda 0}^{(k)}=Q_{\lambda}^{(k)}=\sum_{\substack{\lambda_{1}=0 \\ \lambda_{1}+\lambda_{2}=\lambda}}^{\lambda}(-1)^{\lambda} \frac{\lambda !}{\lambda_{1} ! \lambda_{2} !}\left[(-1)^{\lambda_{1}} A_{2}^{\lambda_{1}} Q_{\lambda_{2}}^{(k)}(1)+A_{1}^{\lambda_{1}} Q_{\lambda_{2}}^{(k)}(2)\right] \frac{R^{\lambda_{1}}}{A^{\lambda_{1}}}
$$

where multipole moments of the DNS nuclei $Q_{\lambda_{2}}^{(k)}(i)(i=1,2)$ are calculated in their centers of mass. For example, up to $\lambda=3$ the values of $Q_{\lambda}^{(k)}$ are:

$$
\begin{aligned}
& Q_{1}^{(m)}=0 \\
& Q_{1}^{(c)}=2 e \frac{A_{2} Z_{1}-A_{1} Z_{2}}{A} R \\
& Q_{2}^{(m)}=2 m_{0} \frac{A_{1} A_{2}}{A} R^{2}+Q_{2}^{(m)}(1)+Q_{2}^{(m)}(2), \\
& Q_{2}^{(c)}=2 e \frac{A_{2}^{2} Z_{1}+A_{1}^{2} Z_{2}}{A^{2}} R^{2}+Q_{2}^{(c)}(1)+Q_{2}^{(c)}(2), \\
& Q_{3}^{(m)}=2 m_{0} \frac{A_{1} A_{2}}{A} \frac{A_{2}-A_{1}}{A} R^{3}+3 \frac{A_{2} Q_{2}^{(m)}(1)-A_{1} Q_{2}^{(m)}(2)}{A} R, \\
& Q_{3}^{(c)}=2 e \frac{A_{2}^{3} Z_{1}-A_{1}^{3} Z_{2}}{A^{3}} R^{3}+3 \frac{A_{2} Q_{2}^{(c)}(1)-A_{1} Q_{2}^{(c)}(2)}{A} R .
\end{aligned}
$$

Here, $A=A_{1}+A_{2}$ and $A_{i}, Z_{i}(i=1,2)$ are the mass number of the system and the mass and charge numbers of the DNS nuclei, respectively; $m_{0}$ is the mass of the nucleon. Experimental values of the quadrupole moments of the DNS nuclei are taken in the calculations. We consider the DNS nuclei in pole-pole orientation which corresponds to the minimum of potential energy. Since the diffuseness in $\rho_{i}^{(k)}(\mathbf{r})$ in the nuclear surface does not practically influence the results for the here considered relative distances $R$, we disregard the effect of diffuseness in the present paper.

The shape of an axially-deformed nucleus can be described by a multipole expansion of the nuclear surface 


$$
R=R_{0}\left(1+\beta_{0} Y_{00}+\beta_{1} Y_{10}+\beta_{2} Y_{20}+\beta_{3} Y_{30}\right)
$$

where $R_{0}$ is the spherical equivalent radius of the nucleus and $\beta_{0}, \beta_{1}, \beta_{2}, \beta_{3}$ are deformation parameters with respect to the center of mass. The $\beta_{\lambda}$ are widely used to characterize the experimental spectroscopic informations. The parameter $\beta_{0}$ is responsible for preserving the nuclear volume. The parameter $\beta_{1}$ provides the vanishing dipole moment $Q_{10}^{(m)}=0$. The deformation parameters $\beta_{2}$ and $\beta_{3}$ are related to the quadrupole and octupole moments of the axially-deformed nucleus. With Eqs. (1) and (5) and assuming a constant nuclear density, we can express the mass multipole moments through the deformation parameters $\beta_{\lambda}(\lambda=0,1,2,3)$ :

$$
\tilde{Q}_{\lambda}^{(m)}=\frac{3}{\lambda+3} A m_{0} R_{0}^{\lambda} \sum_{k_{0}=0}^{\lambda+3} \sum_{k_{1}=0}^{k_{0}} \sum_{k_{2}=0}^{k_{1}} \sum_{k_{3}=0}^{k_{2}} G_{k_{0} k_{1} k_{2} k_{3}}^{\lambda} \beta_{0}^{k_{0}-k_{1}} \beta_{1}^{k_{1}-k_{2}} \beta_{2}^{k_{2}-k_{3}} \beta_{3}^{k_{3}}
$$

where

$$
\begin{aligned}
G_{k_{0} k_{1} k_{2} k_{3}}^{\lambda} & =\frac{1}{2^{\lambda+k_{2}} \lambda !} \sqrt{\frac{3^{k_{1}-k_{2} 5^{k_{2}-k_{3}} 7^{k_{3}}}}{(4 \pi)^{k_{0}}}}\left(\begin{array}{c}
\lambda+3 \\
k_{0}
\end{array}\right)\left(\begin{array}{c}
k_{0} \\
k_{1}
\end{array}\right)\left(\begin{array}{c}
k_{1} \\
k_{2}
\end{array}\right)\left(\begin{array}{c}
k_{2} \\
k_{3}
\end{array}\right) \\
& \times \sum_{i=0}^{k_{2}-k_{3}} \sum_{j=0}^{k_{3}}(-1)^{i+j} 3^{k_{2}-k_{3}-i+j} 5^{k_{3}-j}\left(\begin{array}{c}
k_{2}-k_{3} \\
i
\end{array}\right)\left(\begin{array}{c}
k_{3} \\
j
\end{array}\right) I_{i j}, \\
I_{i j}= & \sum_{k=0}^{[\lambda / 2]}(-1)^{k}\left(\begin{array}{c}
\lambda \\
k
\end{array}\right) \frac{2 \delta_{k_{1}+k_{2}+k_{3}+\lambda+1, o d d}}{(\lambda-2 k) !} \frac{(2 \lambda) !}{\left(k_{1}+k_{2}+k_{3}+\lambda+1-2 i-2 j-2 k\right)} .
\end{aligned}
$$

Here, $\left(\begin{array}{l}n \\ k\end{array}\right)=n ! /(k !(n-k) !)$.

In spite of a better shape parameterization for large deformations [25] one can approximately apply Eq. (5) to the DNS. With Eq. (5) one can well describe the DNS shape for a small mass asymmetry $|\eta|=\left|\left(A_{2}-A_{1}\right) / A\right|<0.5$. For larger $|\eta|$, Eq. (5) leads to smother shapes than the DNS shape. However, even asymmetric configurations can be effectively characterized by the parameters $\beta_{\lambda}$. Using the experimental mass quadrupole and octupole moments, the experimentalists obtain the deformation parameters $\beta_{\lambda}(\lambda=2,3)$ with expressions equal or similar to (6). We do the same procedure but take the DNS multipole moments $Q_{\lambda}^{(m)}$ from (四) instead of moments of SD and HD nuclei, extracted from experiment. Therefore, we solve the system of equations

$$
\tilde{Q}_{\lambda}^{(m)}=Q_{\lambda}^{(m)}
$$

and find the dependences of $\beta_{\lambda}$ on the mass (charge) asymmetry $\eta=\left(A_{2}-A_{1}\right) / A\left(\eta_{Z}=\right.$ $\left.\left(Z_{1}-Z_{2}\right) / Z\right)$ and the relative distance between the centers of nuclei. In Fig. 1 we show the dependence of the parameters $\beta_{1}, \beta_{2}$ and $\beta_{3}$ on the mass asymmetry for the case that the nuclei of the DNS are assumed spherical. This dependence is independent of the total mass number which is simply demonstrated if we take $A_{1}=A(1-\eta) / 2$ and $A_{2}=A(1+\eta) / 2$. A dinuclear system with spherical and equal mass fragments (with radii $R_{0}$ ) in touching leads visually to the axis ratio $2: 1$ because it is the ratio of the DNS length $\left(4 R_{0}\right)$ to the 
DNS width $\left(2 R_{0}\right)$. However, the quadrupole moment $Q_{2}^{(m)}$ for this system is equal to the quadrupole moment of ellipsoid with axis ratio 2.65:1. With deformed nuclei the ratio will be near 3:1 as for hyperdeformed nucleus.

The values of quadrupole and octupole parameters of deformation become close to each other at large mass asymmetry. For very asymmetric DNS consisting of spherical nuclei, we can use simple analytical expressions for $\beta_{2}$ and $\beta_{3}$ :

$$
\begin{array}{r}
\beta_{2}=\sqrt{\frac{5}{4 \pi}} \frac{4 \pi}{3} \frac{A_{1} A_{2}}{A^{2}} \frac{R^{2}}{R_{0}^{2}}, \\
\beta_{3}=\sqrt{\frac{7}{4 \pi}} \frac{4 \pi}{3} \frac{A_{1} A_{2}}{A^{2}} \frac{A_{2}-A_{1}}{A} \frac{R^{3}}{R_{0}^{3}} .
\end{array}
$$

\section{MOMENT OF INERTIA OF DNS}

The moment of inertia is usually calculated microscopically by using the cranking-type formula and taking the residual interactions (for example, the pairing correlations) into consideration. However, one can consider simpler way to find the moment of inertia. The overlap volume of the touching nuclei in the DNS is about a few percent of the total DNS volume and the individuality of the DNS nuclei is conserved. Therefore, one can write down the potential energy $U$ as the sum of binding energies of two nuclei and energy of their interaction (see Sect. 4) and express the multipole moments of the DNS through the multipole moments of the DNS nuclei. Due to the same reason, the DNS moment of inertia $\Im$ can be calculated as

$$
\Im=\Im_{1}+\Im_{2}+m_{0} \frac{A_{1} A_{2}}{A} R_{m}^{2}
$$

where the values of $\Im_{i}(i=1,2)$ are the moment of inertia of the DNS clusters and in the case of small angular momentum are extracted from the experimental value of the energies $E_{2^{+} \rightarrow 0^{+}}$of $2^{+} \rightarrow 0^{+}$transitions: $\Im_{i}=3 / E_{2^{+} \rightarrow 0^{+}}\left(\hbar^{2} / \mathrm{MeV}\right)$ [26].

For large angular momenta, the moments of inertia $\Im_{i}(i=1,2)$ of the DNS nuclei can be with good accuracy calculated in the rigid body approximation

$$
\begin{aligned}
\Im_{i} & =\frac{1}{5} m_{0} A_{i}\left(a_{i}^{2}+b_{i}^{2}\right), \\
a_{i} & =R_{0 i}\left(1-\frac{\alpha_{i}^{2}}{4 \pi}\right)\left(1+\sqrt{\frac{5}{4 \pi}} \alpha_{i}\right), \\
b_{i} & =R_{0 i}\left(1-\frac{\alpha_{i}^{2}}{4 \pi}\right)\left(1-\sqrt{\frac{5}{16 \pi}} \alpha_{i}\right)
\end{aligned}
$$

because the experimental data [28] show that the moment of inertia of superdeformed states is very close to the rigid body limit (about 85 percent of last one). Here, $R_{0 i}$ and $\alpha_{i}$ $(i=1,2)$ are the spherical equivalent radii and the parameters of quadrupole deformation of the DNS nuclei, respectively. The moment of inertia is well measured for the SD and HD states. Therefore, a comparison of calculated and experimental values of the moment of 
inertia can prove our interpretation of the shapes of highly deformed nuclei. Of course, the complete clarification of our cluster approach and the problem how to calculate the moment of inertia could come from attempt to compute actual spectra for direct comparison with data, especially the low-lying negative parity bands symptomatic of octupole deformations. In the forthcoming paper we hope to explain the parity splitting as the effect of tunneling along mass asymmetry (by the analogy with tunneling along $\beta_{3}$ [29]) from the asymmetic DNS with positive $\eta$ to the DNS with the same but negative $\eta$. For this purpose, we need more accurate calculations of the potential energy and, correspondingly, of the moment of inertia as a function of $L$.

\section{POTENTIAL ENERGY OF DNS}

In order to check the possibility of the DNS formation in the excited compound nucleus, the potential energy of the DNS is calculated as [17]

$$
U(R, \eta, L)=B_{1}+B_{2}+V(R, \eta, L)-\left[B_{12}+V_{\text {rot }}^{\prime}(L)\right] .
$$

Here, $B_{1}, B_{2}$, and $B_{12}$ are the realistic binding energies of the fragments and the compound nucleus, respectively. The shell effects are included in these binding energies and supply the local minima in $U(R, \eta, L)$ as a function of $\eta$. The value of $U(R, \eta, L)$ is normalized to the energy of the rotating compound nucleus by $B_{12}+V_{\text {rot }}^{\prime}$. The nucleus-nucleus potential (11)

$$
V(R, \eta, L)=V_{\text {coul }}(R, \eta)+V_{N}(R, \eta)+V_{\text {rot }}(R, \eta, L)
$$

is the sum of the Coulomb 30

$$
V_{\text {coul }}=\frac{Z_{1} Z_{2} e^{2}}{R}+\frac{3}{5} \frac{Z_{1} Z_{2} e^{2}}{R^{3}} \sum_{i=1}^{2} R_{0 i}^{2} \alpha_{i} Y_{20}\left(\cos \theta_{i}\right)+\frac{12}{35} \frac{Z_{1} Z_{2} e^{2}}{R^{3}} \sum_{i=1}^{2} R_{0 i}^{2}\left(\alpha_{i} Y_{20}\left(\cos \theta_{i}\right)\right)^{2}
$$

the centrifugal [17]

$$
V_{\text {rot }}=\frac{\hbar^{2} L(L+1)}{2 \Im}
$$

and the nuclear

$$
V_{N}(R, \eta)=\int \rho_{1}\left(\mathbf{r}_{1}\right) \rho_{2}\left(\mathbf{R}-\mathbf{r}_{2}\right) F\left(\mathbf{r}_{1}-\mathbf{r}_{2}\right) d \mathbf{r}_{1} d \mathbf{r}_{2}
$$

potentials. For the nuclear part of $V(R, \eta, L)$ we use a double folding formalism with the Skyrme-type effective density-dependent nucleon-nucleon interaction

$$
\begin{aligned}
& F\left(\mathbf{r}_{1}-\mathbf{r}_{2}\right)=C_{0}\left(F_{i n} \frac{\rho_{1}\left(\mathbf{r}_{\mathbf{1}}\right)+\rho_{2}\left(\mathbf{R}-\mathbf{r}_{\mathbf{2}}\right)}{\rho_{0}}+F_{e x}\left(1-\frac{\rho_{1}\left(\mathbf{r}_{1}\right)+\rho_{2}\left(\mathbf{R}-\mathbf{r}_{\mathbf{2}}\right)}{\rho_{0}}\right)\right) \delta\left(\mathbf{r}_{1}-\mathbf{r}_{2}\right), \\
& F_{i n, e x}=f_{i n, e x}+f_{i n, e x}^{\prime} \frac{N_{1}-Z_{1}}{A_{1}} \frac{N_{2}-Z_{2}}{A_{2}}
\end{aligned}
$$

which is well known from the theory of finite Fermi systems [17]. Here, $N_{i}(i=1,2)$ are neutron numbers of the nuclei. The values of $C_{0}=300 \mathrm{MeV} \mathrm{fm}{ }^{3}$ and the dimensionless 
parameters $f_{i n}=0.09, f_{e x}=-2.59, f_{i n}^{\prime}=0.42$ and $f_{e x}^{\prime}=0.54$ are fitted to describe a large number of experimental data [31]. For the density of heavy nuclei one can use the two-parameter symmetrized Woods-Saxon function

$$
\rho_{i}(\mathbf{r})=\frac{\rho_{0} \sinh \left(R_{i}\left(\theta_{i}, \phi_{i}\right) / a_{i}\right)}{\cosh \left(R_{i}\left(\theta_{i}, \phi_{i}\right) / a_{i}\right)+\cosh \left(r / a_{i}\right)}
$$

where $\rho_{0}=0.17 \mathrm{fm}^{-3}$ is density in the center of nucleus. We use a nuclear radius parameter $r_{0}=1.14 \mathrm{fm}$ and a diffuseness parameter $a_{i}=(0.54-0.55 \mathrm{fm}$ depending on the mass number of the isotope. For light nuclei with mass numbers $A_{i}<16$, the more realistic functional dependence

$$
\rho_{i}(\mathbf{r})=A_{i}\left(\gamma_{i}^{2} / \pi\right)^{3 / 2} \exp \left(-\gamma_{i}^{2} r^{2}\right)
$$

is used, where $\gamma_{i}$ characterizes the width of the nucleon distribution in the nucleus. The value of $\gamma_{i}$ can be obtained by minimizing the nuclear binding energy in the density function [17]. The deformation effects are taken into account in the calculation of the potential energy surface [17]. The shapes of the DNS nuclei with quadrupole deformations can be written as

$$
R_{i}\left(\theta_{i}, \phi_{i}\right)=R_{0 i}\left(1-\frac{\alpha_{i}^{2}}{4 \pi}+\alpha_{i} Y_{20}\left(\theta_{i}, \phi_{i}\right)\right)
$$

With the nucleon-nucleon interaction (16) the repulsive core appears in $V_{N}(R, \eta)$ which prevents the motion to smaller distances $\left(R<R_{1}+R_{2}\right)$ and reflects the action of the Pauli principle. For $R_{1}+R_{2}-1 \mathrm{fm}<R<R_{1}+R_{2}+2 \mathrm{fm}$ the potential $V(R, \eta, L)$ as a function of the relative distance $R$ has a pocket with small depth which is resulted by the atractive nuclear and repulsive Coulomb interactions. The DNS is localized in the minimum of this pocket at $R_{m} \approx R_{1}+R_{2}=R_{01}\left(1+\sqrt{\frac{5}{4 \pi}} \alpha_{1}\right)+R_{02}\left(1+\sqrt{\frac{5}{4 \pi}} \alpha_{2}\right)$ for the pole-pole configuration. The relative orientation of the deformed nuclei in the DNS follows the minimum of the potential energy which yields the pole-pole orientation. At fixed mass asymmetry $\eta$, charge asymmetry $\eta_{Z}$ and deformation parameters $\alpha_{i}(i=1,2)$ the minimum of $V(R, \eta, L)$ of the DNS corresponds to the distance $R=R_{m}\left(\eta, \eta_{Z}, \alpha_{i}\right)$ when the poles of nuclei touch each other. For each $\eta$, we minimize $U\left(R_{m}, \eta, L\right)$ with respect to the charge asymmetry. Since the minimization of $U$ with respect to $\alpha_{i}$ ia very cumbersome, we took from Refs. [26,27] the parameters of the quadrupole deformation which are related to the excitation of the first $2^{+}$states if the energies of these states are smaller than $1.5 \mathrm{MeV}$. As is known from experiments on sub-barrier fusion, these states are easily populated. For the treatment of small internal excitation of the DNS nuclei, this approximation seems to be good for deriving the minimum of $U$ with respect to $\alpha_{i}$. So, the energy $U\left(R_{m}, \eta, L\right)$ plotted as a function of $\eta$ results minimum values $B_{1}+B_{2}+V(R, \eta, L)$ with respect to other degrees of freedom in the DNS.

The potentials for all DNS were calculated with the same set of parameters and assumptions. We found that the final results are not crucial to a reasonable variation of these parameters in the calculation of the potential energy. Since the excitation energies of the DNS nuclei are relatively small, the calculation of the DNS potential energy immediately shows which DNS configuration can be related to the SD or HD nuclear systems. Among all minima in $U\left(R_{m}, \eta, L\right)$ as a function of $\eta$ we selected those with the minimal values of 
$U$. These minima appear for small and large $\eta$ and we analysed either almost symmetric or very asymmetric configurations. If there are several minima with close energies in some interval of $\eta$, all of them are treated. However, the calculated values of $Q_{2}$ and $\Im$ would be similar for these DNS.

\section{RESULTS AND DISCUSSIONS}

With the formalism given in Sections II-IV we studied various nuclei described with the dinuclear system concept. As compound nuclei we chose ${ }^{152} \mathrm{Dy},{ }^{232} \mathrm{Th},{ }^{234} \mathrm{U},{ }^{240} \mathrm{Pu},{ }^{76} \mathrm{Kr}$, ${ }^{148} \mathrm{Nd}$ and ${ }^{236} \mathrm{Ra}$ which will be discussed next.

a) ${ }^{152}$ Dy:

The dependences of $\beta_{2}, \beta_{3}, Q_{2}\left(Q_{2}=Q_{2}^{(c)}\right)$ and $Q_{3}\left(Q_{3}=Q_{3}^{(c)}\right)$ on the mass asymmetry $\eta$ are presented in Fig. 2 for the DNS corresponding to the ${ }^{152} \mathrm{Dy}$ compound nucleus. Since the deformations of the DNS nuclei are functions of $\eta$, these dependences have some oscillations. While $Q_{2}$ and $\beta_{2}$ decrease with increasing $\eta, Q_{3}$ and $\beta_{3}$ have maxima. The positions of these maxima approximately correspond to maximal potential energy of the DNS as a function of $\eta$ (see Fig. 3). The value of $\beta_{3}$ increases quickly with $\eta$ from zero. For very asymmetric DNS, $\beta_{3}$ becomes again relatively small. Therefore, a given value of $\beta_{3}$ can correspond to two different DNS with different mass asymmetries. For the symmetrical DNS with spherical nuclei, the difference by a factor $\sqrt{2}$ between our value of $\beta_{2}$ and $\beta_{2}$ obtained in [22] is due to another definition of $\beta_{2}$ in [22].

The DNS potential energy as a function of the mass asymmetry is shown in Fig. 3 for the cases of spherical and deformed nuclei in the DNS forming ${ }^{152} \mathrm{Dy}$. The calculation with deformed nuclei yields various minima of the potential energy, especially at $\eta=0.026$ $\left({ }^{74} \mathrm{Ge}+{ }^{78} \mathrm{Se}\right), \eta=0.16\left({ }^{64} \mathrm{Ni}+{ }^{88} \mathrm{Sr}\right)$ and $\eta=0.34\left({ }^{50} \mathrm{Ti}+{ }^{102} \mathrm{Ru}\right)$. For zero angular momentum, the energy of the combination ${ }^{50} \mathrm{Ti}+{ }^{102} \mathrm{Ru}$ is about $20 \mathrm{MeV}$ which is close to the value estimated for the HD state in [32]. We calculated the DNS moment of inertia $\Im$ using the experimental moments $\Im_{i}$ of the DNS nuclei $\left(\Im=\Im^{e}\right)$ or the rigid body moments $\Im_{i}$ given in eq.(10) $\left(\Im=\Im^{r}\right)$. The first value of the moment of inertia of the system ${ }^{50} \mathrm{Ti}+{ }^{102} \mathrm{Ru}$ is obtained as $\Im^{e}=100 \hbar^{2} \mathrm{MeV}^{-1}$ and the second one as $\Im^{r}=131 \hbar^{2} \mathrm{MeV}^{-1}$. The latter value is close to the experimental one $\Im^{e x p}=130 \hbar^{2} \mathrm{MeV}^{-1}$ [2]. For this DNS, the obtained value $\beta_{2}=1.3$ is in agreement with the experimental estimate $\beta_{2} \geq 0.9$. Therefore, we conclude that the shape of the DNS ${ }^{50} \mathrm{Ti}+{ }^{102} \mathrm{Ru}$ is compatible with the shape of the ${ }^{152} \mathrm{Dy}$ nucleus in the HD state.

We found that in the asymmetric DNS, for example ${ }^{22} \mathrm{Ne}+{ }^{130} \mathrm{Ba} \quad(\eta=0.71)$ and ${ }^{26} \mathrm{Mg}+{ }^{126} \mathrm{Xe}(\eta=0.66)$ (where the potential energy has minima), the calculated moments of inertia and quadrupole moments are close to the experimental values $\Im^{\text {exp }}=(85 \pm$ $3) \hbar^{2} \mathrm{MeV}^{-1}$ and $Q_{2}^{e x p}=(18 \pm 3) e$ barn, respectively, known for the SD state. For the system ${ }^{22} \mathrm{Ne}+{ }^{130} \mathrm{Ba}$, we calculated $\Im^{r}=100 \hbar^{2} \mathrm{MeV}^{-1}$ (and $\Im^{e}=63 \hbar^{2} \mathrm{MeV}^{-1}$ ), $Q_{2}=20 e$

barn and $\beta_{2}=0.8$. In the system ${ }^{26} \mathrm{Mg}+{ }^{126} \mathrm{Xe}$ we obtained $\Im^{r}=104 \hbar^{2} \mathrm{MeV}^{-1}$ (and $\left.\Im^{e}=67 \hbar^{2} \mathrm{MeV}^{-1}\right), Q_{2}=24 e$ barn and $\beta_{2}=0.9$. These DNS have practically zero temperature if they are formed in the reaction ${ }^{48} \mathrm{Ca}(205 \mathrm{MeV})\left({ }^{108} \mathrm{Pd}, 4 \mathrm{n}\right){ }^{152} \mathrm{Dy}$. At $L=0$ the potential energies of these DNS with respect to the ground state of ${ }^{152}$ Dy lie about $8 \mathrm{MeV}$ higher than those estimated for the SD shapes in [32]. The energies of these two systems 
with respect to the energy of the compound nucleus are shown in Fig. 4 as a function of the square of the angular momentum.

The SD bands in the mass region $A \approx 150$ are populated with an anomalously high intensity around a spin of $55 \hbar$. There is no significant population of these states below $(45-50) \hbar$ 33. This fact can be explained by the DNS interpretation of the SD states. The dependence of the DNS potential energy on $\eta$ becomes flat with decreasing angular momentum and the DNS can evolve to larger $\eta$. This takes place because the potential barrier in the direction of larger mass asymmetries decreases with $L$ (Fig. 3). Due to the distribution of the excitation energy among many configurations at small $L$ and due to the motion of the DNS to the compound nucleus with increasing $\eta$, the transition from the SD state to the ground state is invisible.

b) ${ }^{232} \mathrm{Th}$ :

Analysing the potential energy of the DNS as a function of $\eta$ for the ${ }^{232} \mathrm{Th}$ compound nucleus (see Fig. 5), we found well exposed minima corresponding to the systems ${ }^{100} \mathrm{Zr}+{ }^{132} \mathrm{Sn}$ $(\eta=0.138)$ and ${ }^{82} \mathrm{Ge}+{ }^{150} \mathrm{Ce}(\eta=0.293)$. Fig. 6 shows the dependence of $\beta_{2}$ and $\Im=\Im^{r}$ on $\eta$ which is weak for small mass asymmetries. In both ${ }^{232} \mathrm{Th}$ systems, $\beta_{2}$ is about 1.5 , $\beta_{3} \approx 0.40, \Im^{r}$ about $290 \hbar^{2} \mathrm{MeV}^{-1}$ and $\Im^{e} \approx 240 \hbar^{2} \mathrm{MeV}^{-1}$; the potential energies, given in Fig. 5, are near the energy of the ground state of ${ }^{232} \mathrm{Th}$. Excepting the values of $\beta_{2}$, the obtained values in Table 1 are close to the corresponding ones calculated for the third minimum in [20]. In our calculation $\beta_{2}$ is larger in comparison to $\beta_{2}=0.85$ in [20]. The $\mathrm{SD}$ rotational bands in ${ }^{232} \mathrm{Th}$ can be interpreted as the DNS states of the configurations ${ }^{28} \mathrm{Mg}+{ }^{204} \mathrm{Pt}$ and ${ }^{26} \mathrm{Ne}+{ }^{206} \mathrm{Hg}$ (Table 1).

c) ${ }^{234} \mathrm{U}$ and ${ }^{240} \mathrm{Pu}$ :

For the ${ }^{234} \mathrm{U}$ nucleus, we found the $\mathrm{SD}$ and $\mathrm{HD}$ cluster configurations (Table 1): ${ }^{26} \mathrm{Ne}+{ }^{208} \mathrm{~Pb},{ }^{28} \mathrm{Mg}+{ }^{206} \mathrm{Hg},{ }^{82} \mathrm{Ge}+{ }^{152} \mathrm{Nd},{ }^{100} \mathrm{Zr}+{ }^{134} \mathrm{Te}$ and ${ }^{104} \mathrm{Mo}+{ }^{130} \mathrm{Sn}$. The experimentally extracted depth of the third well (which corresponds to the HD state) was determined to be $(3.6 \pm 0.3) \mathrm{MeV}$ for the ${ }^{234} \mathrm{U}$ nucleus [34]. For the DNS configurations, this value is an agreement with the value of the depth of the pocket in the nucleus-nucleus potential as function of $\eta$. For example, for the ${ }^{100} \mathrm{Zr}+{ }^{134} \mathrm{Te}$ and ${ }^{104} \mathrm{Mo}+{ }^{130} \mathrm{Sn}$ configurations, the depth of the pocket is about $3.4 \mathrm{MeV}$.

Fig. 5 shows also the potential energy of the ${ }^{240} \mathrm{Pu}$ compound nucleus where SD bands were observed in an experiment on sub-barrier fission [34]. These SD states could be considered as the DNS-type states: ${ }^{32} \mathrm{Mg}+{ }^{208} \mathrm{~Pb}$ and ${ }^{34} \mathrm{Si}+{ }^{206} \mathrm{Hg}$ (see Table 1). From the results presented in Table 1 one can predict some HD cluster states for the ${ }^{240} \mathrm{Pu}$ nucleus.

d) ${ }^{74} \mathrm{Kr}$ and ${ }^{72} \mathrm{Se}$ :

The potential energy of the DNS for the ${ }^{76} \mathrm{Kr}$ compound nucleus is shown in Fig. 5. We find a deep minimum with an energy on the level of the energy of the compound nucleus for the cluster configuration ${ }^{8} \mathrm{Be}+{ }^{68} \mathrm{Ge}$. For this cluster state we have $Q_{2}=4.9 \mathrm{e}$ barn, $Q_{3}=2.0 \times 10^{3} e \mathrm{fm}^{3}$ and $\Im^{r}=29 \hbar^{2} / \mathrm{MeV}\left(\Im^{e}=17.2 \hbar^{2} / \mathrm{MeV}\right)$. A similar picture is observed in other nuclei in the $A \approx 76$ mass region, for example, in ${ }^{74} \mathrm{Kr}$ and ${ }^{72} \mathrm{Se}$. In the DNS configuration with the light cluster ${ }^{8} \mathrm{Be}$ we found $Q_{2}=4.7 \mathrm{e}$ barn, $Q_{3}=1.9 \times 10^{3} \mathrm{e} \mathrm{fm}^{3}$ for the ${ }^{72} \mathrm{Se}$ compound nucleus. For the nuclei in the mass region $A \approx 100$, the energies of such cluster configurations are by 5 to $6 \mathrm{MeV}$ higher than the energies of corresponding compound nuclei.

Let us consider the $N / Z$-equilibrium. In the nuclei ${ }^{72} \mathrm{Se},{ }^{74,76} \mathrm{Kr}$ we obtained minima in 
the DNS potential energy for configurations with $\alpha$-particle nuclei (multiple of $\alpha$-clusters) at large $\eta$. The potential energies of these DNS are small because the light nuclei in them are most stable. Such behaviour of the DNS potential energy is not observed for asymmetric configurations with light clusters in the $A \approx 100$ mass region.

e) $\alpha$-structure and dipole and octupole deformations, ${ }^{148} \mathrm{Nd},{ }^{226} \mathrm{Ra}$ and ${ }^{220-228} \mathrm{Th}$ :

If very asymmetric DNS are energetically favorable, the wave function of the compound nucleus has components belonging to cluster-type configurations. For example, in many cases the cluster configurations

$$
{ }^{A} Z \rightarrow{ }^{(A-4)}(Z-2)+{ }^{4} \mathrm{He}
$$

have an energy which is close or even lower than the energy of the ground state of the compound nucleus. As a result, the nucleus can have an octupole deformation in the ground state. Since the shape of the nucleus is no more symmetric under space inversion, the spectrum of such a nucleus must contain states with different parity. This was experimentally found in many nuclei with $Z \approx 88-90(N \approx 86-90)$, in different isotopes of the nuclei $\mathrm{Ra}$, Th and $\mathrm{U}$, and with $Z \approx 60$, in isotopes of $\mathrm{Ba}, \mathrm{Ce}, \mathrm{Nd}, \mathrm{Sm}$ and $\mathrm{Gd}$ [35]. Another consequence of the asymmetric shape is the appearance of E1 and E3 transitions. These transitions were found in ${ }^{226} \mathrm{Ra}$ with $Q_{2}=750 e \mathrm{fm}^{2}, Q_{3}=3100 e \mathrm{fm}^{3}$ 37 and in ${ }^{148} \mathrm{Nd}$ with $Q_{2}=400 e \mathrm{fm}^{2}, Q_{3}=1500 \mathrm{efm}^{3}[38]$. The experimental dipole moments of this nuclei are found to be $0.16 e \mathrm{fm}$ for ${ }^{226} \mathrm{Ra}$ [37] and $0.32 e$ fm for ${ }^{148} \mathrm{Nd}$ [38]. Under the assumption that the cluster configuration (20) mainly contributes to the ground state with a static octupole deformation, we can obtain the values of the multipole moments for these nuclei. For ${ }^{226} \mathrm{Ra}$, we found $Q_{1}=Q_{1}^{(c)} / 2=4 e \mathrm{fm}, Q_{2}=776 e \mathrm{fm}^{2}, Q_{3}=2662 e \mathrm{fm}^{3}$. For ${ }^{148} \mathrm{Nd}$, we obtained $Q_{1}=3 e \mathrm{fm}, Q_{2}=486 e \mathrm{fm}^{2}, Q_{3}=1844 e \mathrm{fm}^{3}$. The calculated values of $Q_{2}$ and $Q_{3}$ are close to the experimental ones mentioned before. However, the values of $Q_{1}$ are larger by at least one order of magnitude. The same problem was observed in the cluster model [36]. These deviations of the theoretical dipole moments from the experimental ones are due to the use of a simplified treatment of the $N_{1} / Z_{1}$-ratios in the DNS nuclei. The value of $Q_{1}$ strongly depends on these ratios and vanishes $\left(Q_{1}=0\right)$ in the limit of the same $N / Z$-ratio in the DNS nuclei. For very asymmetric DNS, the $N / Z$-ratios in the light nucleus are effectively larger than the $N / Z$-ratio in the $\alpha$-particle $(N / Z=1)$ because the heavy nucleus of the DNS is strongly overlapping with the $\alpha$-particle and there is at least one valence neutron supplying the coupling of the $\alpha$-particle with the heavy cluster. If we take the $\left({ }^{4} \mathrm{He}+1 n\right)$ cluster instead of ${ }^{4} \mathrm{He}$ in the DNS or slightly increase the $N / Z$-ratio in the contact region of the two nuclei of the DNS, then the theoretical value of $Q_{1}$ for ${ }^{226} \mathrm{Ra}$ and ${ }^{148} \mathrm{Nd}$ results in agreement with the experimental data, but with $Q_{2}$ and $Q_{3}$ practically not changed. For the nuclei with octupole deformation in the ground state, one can try to explain the parity splitting as a function of $L$ by the tunneling in $\eta$ in the DNS with $\alpha$-particle.

As shown in Fig. 7, the octupole (quadrupole) deformation becomes smaller (larger) with an increasing atomic number of the Th isotopes in the systems ${ }^{(A-4)} \mathrm{Ra}+{ }^{4} \mathrm{He}$. This can be explained by the fact that the quadrupole deformation of the heavy cluster of the DNS gets larger in this mass region. Such a behaviour of $\beta_{2}$ and $\beta_{3}$ is in agreement with the one obtained in 39 . 


\section{SUMMARY}

The DNS potential energy as a function of mass asymmetry has a few global minima. Most of them lie higher in energy than the energy of the compound nucleus. It is possible, however, to populate these states in heavy ion induced reactions by choosing appropriate reaction partners with an appropriate bombarding energy. At high spins, these cluster states can be cold and have long lifetime. We found the energies, moments of inertia and quadrupole deformations of certain DNS close to the experimental ones of SD and HD nuclei. Since many DNS states have relatively large octupole deformations, the experimental measurement of the octupole deformation of highly deformed nuclei can answer the question whether these nuclei exist in cluster configurations. An evidence, that SD nuclei can have octupole deformations, is for example an observation of an excited SD band in ${ }^{190} \mathrm{Hg}$ which decays to the lowest-energy (yrast) SD-band by transitions of odd multipolarity. The E1 rate observed in [40] is three orders of magnitude larger then those rates typically observed in heavy deformed nuclei and is similar to those observed in octupole-unstable normallydeformed actinide nuclei 41.

In some nuclei with $A \approx 230$ or $A \approx 76$ the potential energy of the DNS has minima which lie roughly on the same energy as the energy of the compound nucleus. This means that such cluster states can exist at low spins. We attempted to describe the nuclei with static octupole deformation in the ground state like the DNS where the $\alpha$ - cluster is the lighter cluster. It was found that calculated quadrupole and octupole deformations are close to the experimental ones and that such DNS are energetically favorable.

\section{ACKNOWLEDGMENTS}

We thank Prof. R.V.Jolos for fruitful discussions and Yu.V.Palchikov for valuable advices regarding the calculations. G.G.A. and T.M.S. are grateful the Alexander von Humboldt-

Stiftung and the European Physical Society for support, respectively. This work was supported in part by DFG and RFBR. 


\section{REFERENCES}

[1] P.J. Twin et al., Phys. Rev. Lett. 57 (1986) 811.

[2] A. Galindo-Uribarri et al., Phys. Rev. Lett. 71 (1993) 231.

[3] S.M. Polikanov, JETP. 15 (1962) 1016.

[4] H.J. Specht, Phys. Lett. B41 (1972) 43.

[5] J.H. Hamilton et al., Phys. Rev. Lett. 32 (1974) 239.

[6] R.B. Piercey, Phys. Rev. Lett. 47 (1981) 1514.

[7] T.A. Kahn et al., Z. Phys. A283 (1977) 103.

[8] R.F. Azuma et al., Phys. Lett. B86 (1979) 5.

[9] J.H. Hamilton, Prog. Part. Nucl. Phys. 28 (1992) 87.

[10] B.B. Brick et al., Phys. Rev. Lett. 28 (1972) 1707.

[11] K.Th. Brinkmann et al., Nucl. Phys. A502 (1989) 271.

[12] S. Aberg et al., Annu. Rev. Nucl. Part. Sci. 40 (1990) 439.

[13] R.V.F. Janssens et al., Annu. Rev. Nucl. Part. Sci. 41 (1991) 321.

[14] J. Dudek et al. Phys. Lett. B211 (1988) 252.

[15] P. Ring and A.V. Afanasjev, Prog. Part. Nucl. Phys. 38 (1997) 137.

[16] N. Cindro, J. Phys. G4 (1978) L23; N. Cindro and W. Greiner, J. Phys. G9 (1983) L175.

[17] G.G. Adamian et al., Int. J. Mod. Phys. E5 (1996) 191.

[18] Yu.V. Pyatkov et al., Nucl. Phys. A624 (1997) 140.

[19] S. Aberg, Z. Phys. A349 (1994) 205.

[20] S. Cwiok et al., Phys. Lett. B322 (1994) 304.

[21] B. Buck, A.C. Merchant and S.M. Perez, Phys. Rev. Lett. 65 (1990) 2975; Phys. Rev. Lett. 76 (1996) 380; Phys. Rev. C58 (1998) 2049.

[22] S. Royer, J. Phys. G21 (1995) 339.

[23] Yu.F. Smirnov and Yu.M. Tchuvil'sky, Phys.Lett. B314 (1984) 25.

[24] G.G. Adamian, N.V. Antonenko and Yu.M. Tchuvil'sky, Phys. Lett. B451 (1999) 289.

[25] V.V. Pashkevich, Nucl. Phys. A169 (1971) 275.

[26] S. Raman et al., At. Data Nucl. Data Tables 36 (1987) 1.

[27] P. Möller, R. Nix, At. Data and Nucl. Tables 39 (1988) 213; http://t2.lanl.gov/data/ astro/molnix96/massd.html.

[28] J. Dudek, Prog. Part. Nucl. Phys. 28 (1992) 131.

[29] R.V. Jolos and P. von Brentano, Phys. Rev C49 (1994) R2301; Nucl. Phys. A587 (1995) 377.

[30] C.Y. Wong, Phys. Rev. Lett. 31 (1973) 766.

[31] A. B. Migdal, Theory of Finite Fermi Systems and Application to Atomic Nuclei (Nauka, Moscow, 1982).

[32] S. Aberg, Nucl. Phys. A557 (1993) 17.

[33] M.A. Bentley et al., J. Phys. G17 (1991) 481.

[34] D. Gassmann et al., Verhandl. DPG (VI) 34 (1999) 162; A. Krasznahorkay et al., Phys. Lett. B461 (1999) 15; Phys. Rev. Lett. 80 (1998) 2073.

[35] P.A. Butler and W. Nazarewicz, Rev. Mod. Phys. 68 (1996) 350.

[36] B. Buck, A.C. Merchant and S.M. Perez, Phys. Rev. C59 (1999) 750.

[37] H.J. Wollersheim, Nucl. Phys. A556 (1993) 261.

[38] R. Ibbotson, Phys. Rev. Lett. 71 (1993) 1990. 
[39] W. Nazarewicz et al., Nucl. Phys. A467 (1987) 437.

[40] B. Crowell et al., Phys. Lett. B333 (1993) 320.

[41] I. Ahmad and P.A. Butler, Ann. Rev. Nucl. Part. Sci. 43 (1993) 71. 


\section{TABLES}

TABLE I. The values of moments of inertia $\Im^{r}$ and $\Im^{e}$, charge quadrupole moments $Q_{2}$ and octupole moments $Q_{3}$, quadrupole and octupole deformation parameters $\beta_{2}$ and $\beta_{3}$ for different DNS corresponding to the compound nuclei ${ }^{232} \mathrm{Th},{ }^{234} \mathrm{U},{ }^{240} \mathrm{Pu}$ (see text).

\begin{tabular}{c|c|c|c|c|c|c|}
\hline \hline Clust. Conf. & $\Im^{r}\left(\hbar^{2} / \mathrm{MeV}\right)$ & $\Im^{e}\left(\hbar^{2} / \mathrm{MeV}\right)$ & $Q_{2} \times 10^{2}\left(e \mathrm{fm}^{2}\right)$ & $Q_{3} \times 10^{3}\left(e \mathrm{fm}^{3}\right)$ & $\beta_{2}$ & $\beta_{3}$ \\
\hline${ }^{26} \mathrm{Ne}+{ }^{206} \mathrm{Hg} \rightarrow{ }^{232} \mathrm{Th}$ & 171 & 82 & 24.9 & 18.8 & 0.57 & 0.63 \\
${ }^{28} \mathrm{Mg}+{ }^{204} \mathrm{Pt} \rightarrow{ }^{232} \mathrm{Th}$ & 180 & 94 & 31.0 & 23.9 & 0.65 & 0.68 \\
${ }^{82} \mathrm{Ge}+{ }^{150} \mathrm{Ce} \rightarrow{ }^{232} \mathrm{Th}$ & 292 & 249 & 70.9 & 19.8 & 1.53 & 0.47 \\
${ }^{100} \mathrm{Zr}+{ }^{132} \mathrm{Sn} \rightarrow{ }^{232} \mathrm{Th}$ & 292 & 235 & 70.1 & 16.2 & 1.48 & 0.34 \\
\hline${ }^{26} \mathrm{Ne}+{ }^{208} \mathrm{~Pb} \rightarrow{ }^{234} \mathrm{U}$ & 169 & 79 & 20.9 & 18.0 & 0.47 & 0.61 \\
${ }^{28} \mathrm{Mg}+{ }^{206} \mathrm{Hg} \rightarrow{ }^{234} \mathrm{U}$ & 179 & 91 & 29.9 & 23.7 & 0.61 & 0.68 \\
${ }^{82} \mathrm{Ge}+{ }^{152} \mathrm{Nd} \rightarrow{ }^{234} \mathrm{U}$ & 291 & 257 & 70.4 & 20.5 & 1.49 & 0.49 \\
${ }^{100} \mathrm{Zr}+{ }^{134} \mathrm{Te} \rightarrow{ }^{234} \mathrm{U}$ & 326 & 258 & 71.8 & 14.0 & 1.73 & 0.27 \\
${ }^{104} \mathrm{Mo}+{ }^{130} \mathrm{Sn} \rightarrow{ }^{234} \mathrm{U}$ & 296 & 242 & 71.8 & 14.0 & 1.48 & 0.28 \\
\hline${ }^{32} \mathrm{Mg}+{ }^{208} \mathrm{~Pb} \rightarrow{ }^{240} \mathrm{Pu}$ & 191 & 101 & 28.7 & 23.2 & 0.57 & 0.71 \\
${ }^{34} \mathrm{Si}+{ }^{206} \mathrm{Hg} \rightarrow{ }^{240} \mathrm{Pu}$ & 197 & 107 & 33.9 & 25.4 & 0.69 & 0.70 \\
${ }^{82} \mathrm{Ge}+{ }^{158} \mathrm{Sm} \rightarrow{ }^{240} \mathrm{Pu}$ & 307 & 267 & 75.3 & 22.1 & 1.53 & 0.50 \\
${ }^{104} \mathrm{Zr}+{ }^{136} \mathrm{Xe} \rightarrow{ }^{240} \mathrm{Pu}$ & 305 & 253 & 74.1 & 14.8 & 1.49 & 0.33 \\
${ }^{106} \mathrm{Mo}+{ }^{134} \mathrm{Te} \rightarrow{ }^{240} \mathrm{Pu}$ & 314 & 258 & 76.4 & 16.0 & 1.52 & 0.32 \\
${ }^{110} \mathrm{Ru}+{ }^{130} \mathrm{Sn} \rightarrow{ }^{240} \mathrm{Pu}$ & 311 & 250 & 75.3 & 12.4 & 1.49 & 0.23 \\
\hline \hline
\end{tabular}




\section{FIGURES}

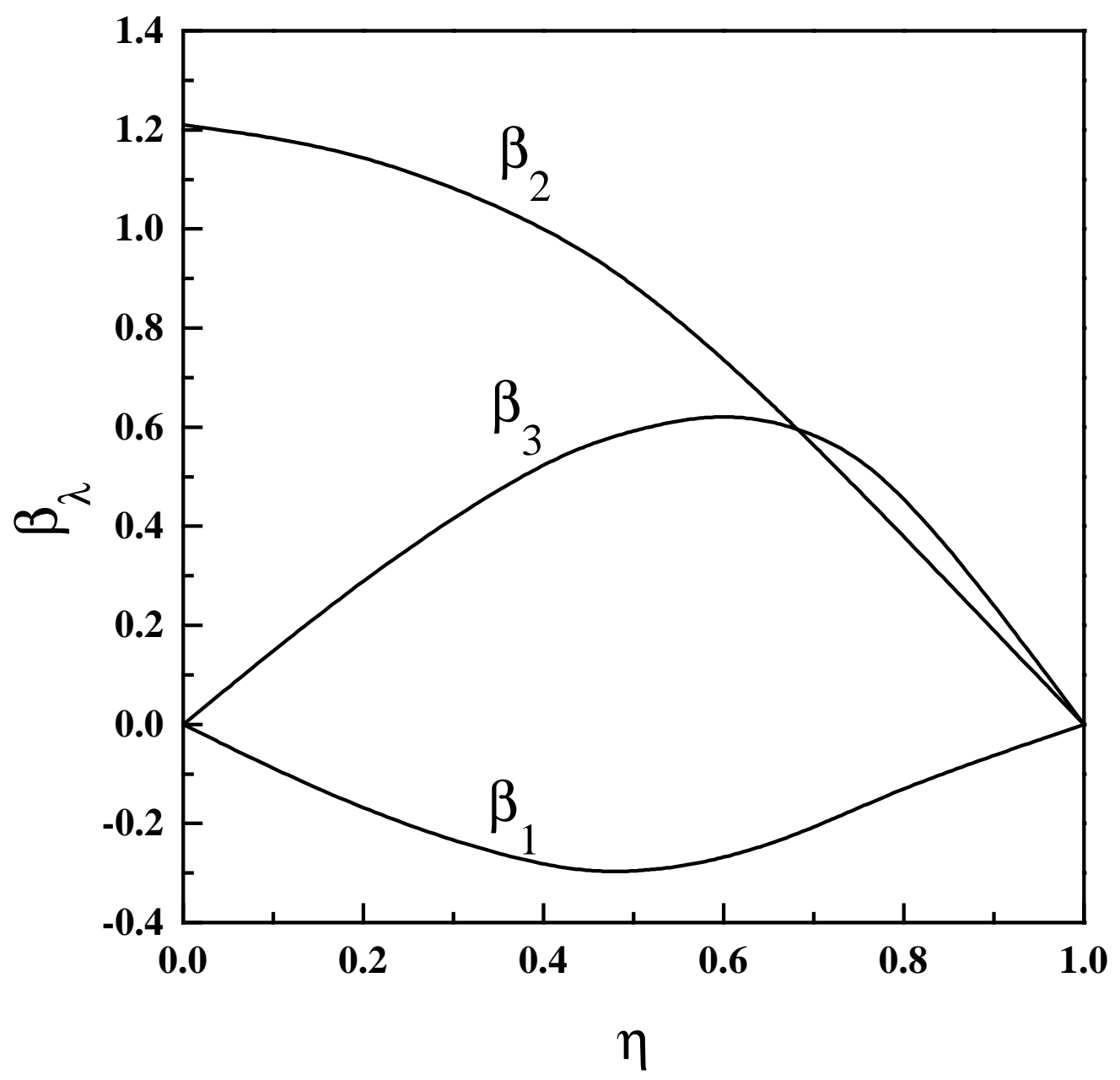

FIG. 1. Dependence of the deformation parameters $\beta_{1}, \beta_{2}, \beta_{3}$ on mass asymmetry $\eta$ for a DNS with spherical nuclei. The parameters do not depend on the total mass number of the DNS. 


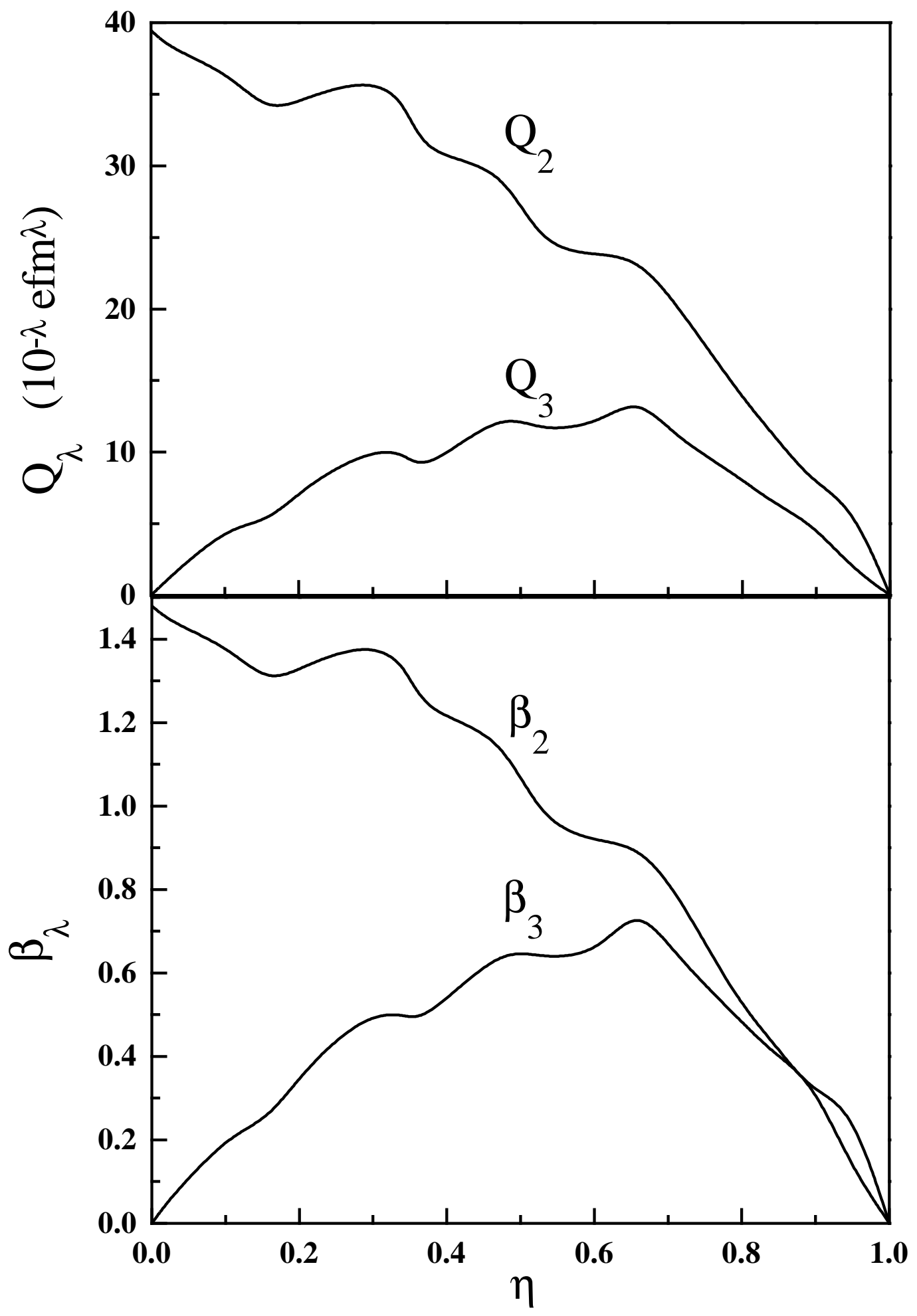

FIG. 2. Dependence of $Q_{2}\left(10^{-2} e \mathrm{fm}^{2}\right)$ and $Q_{3}\left(10^{-3} e \mathrm{fm}^{3}\right)$ (upper part) and of $\beta_{2}$ and $\beta_{3}$ (lower part) on the mass asymmetry $\eta$ of the DNS for the ${ }^{152}$ Dy compound nucleus. The deformation of the DNS nuclei are taken into account. 


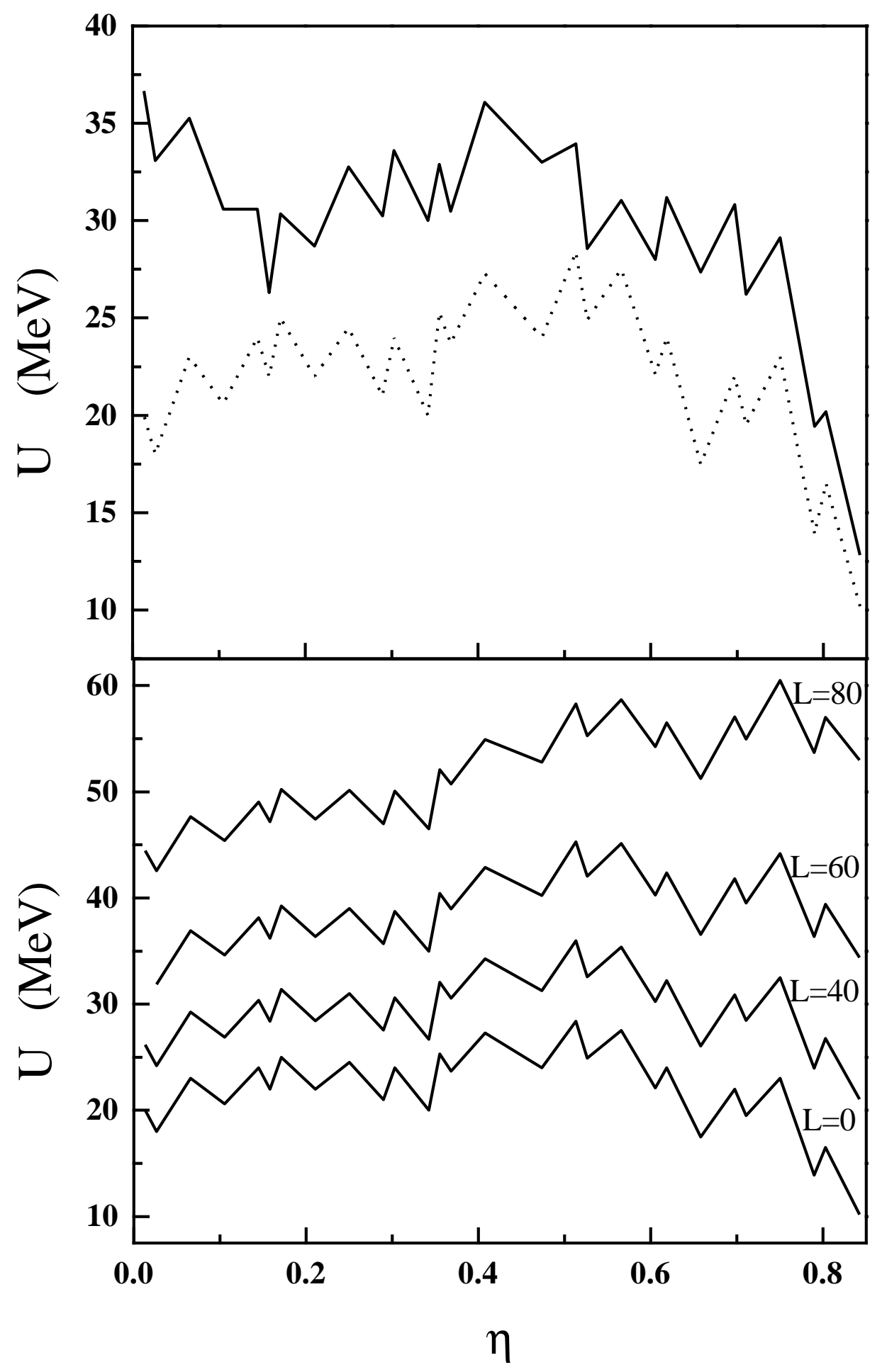

FIG. 3. Potential energy $U$ of the DNS as a function of mass asymmetry $\eta$ for the ${ }^{152}$ Dy compound nucleus. At $L=0$ the calculated results without and with deformation of the DNS nuclei are presented by solid and dotted lines, respectively (upper part). The lower part shows results for different angular momentum quantum numbers calculated with deformed DNS nuclei. 


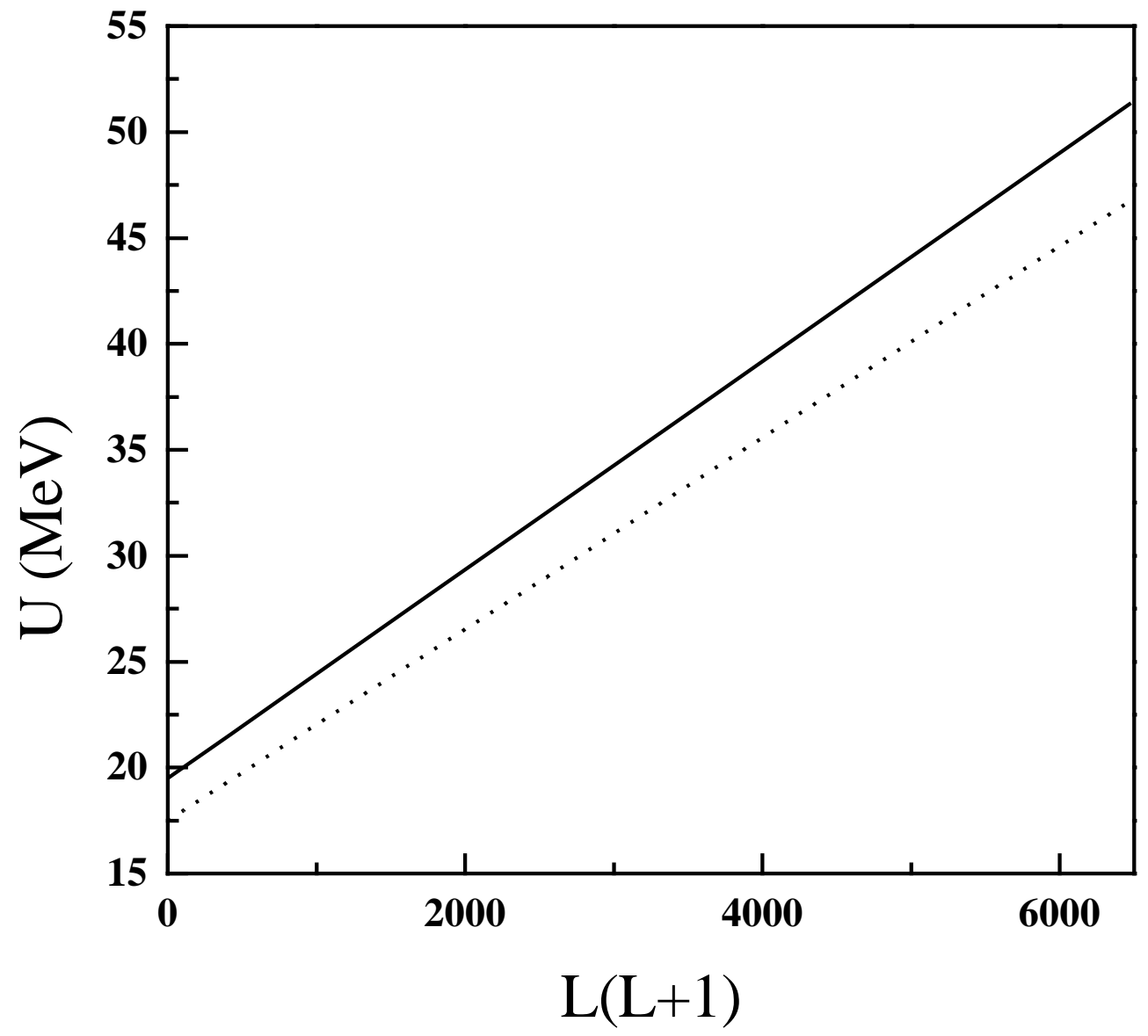

FIG. 4. Dependence of the energies of the systems ${ }^{22} \mathrm{Ne}+{ }^{130} \mathrm{Ba}$ (dotted line) and ${ }^{26} \mathrm{Mg}+{ }^{126} \mathrm{Xe}$ (solid line) on the square of the angular momentum quantum number. The energy is normalized to the energy of the rotating compound nucleus. 


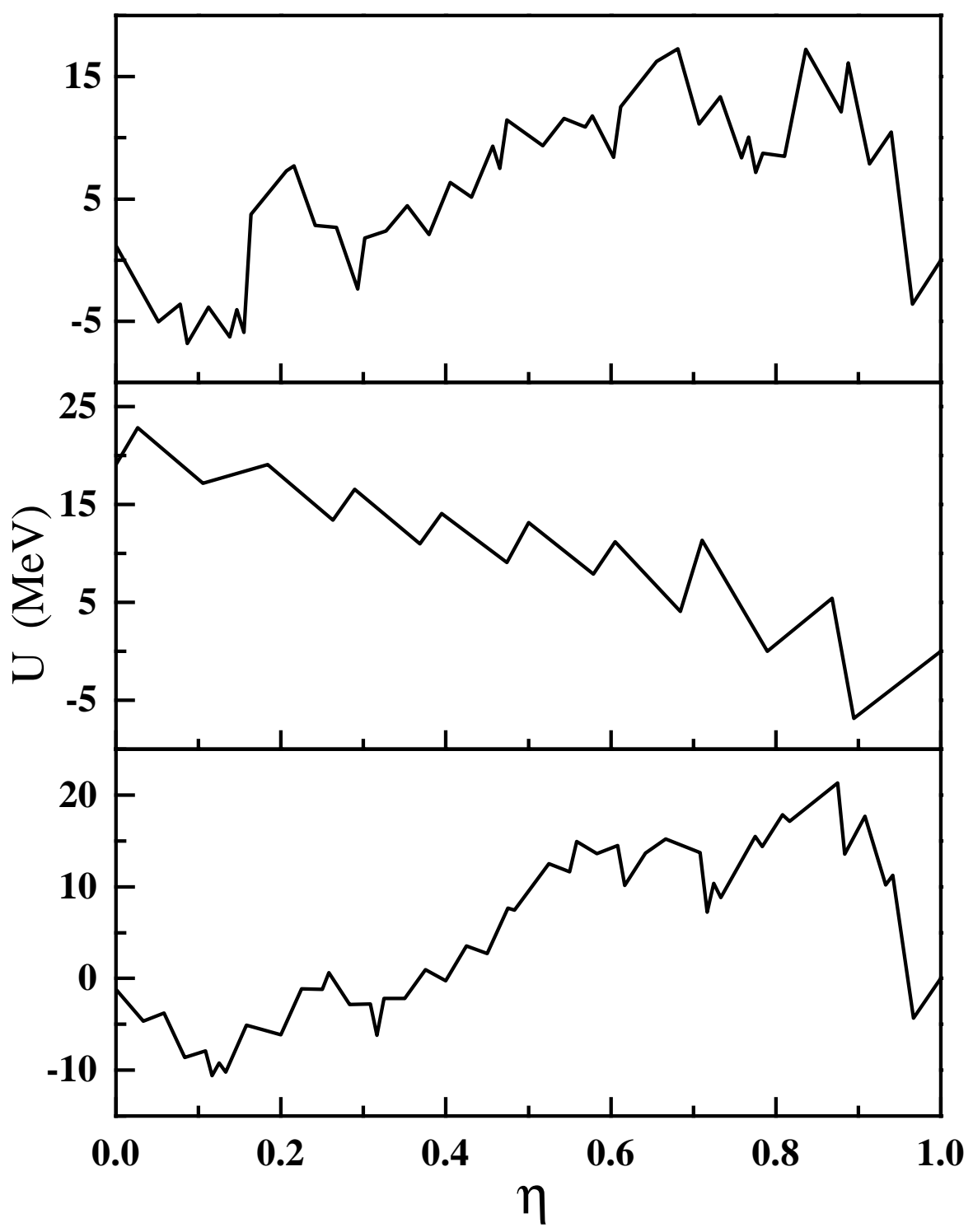

FIG. 5. Potential energy $U$ of the DNS as a function of $\eta$ for the compound nuclei ${ }^{232} \mathrm{Th}$ (upper part), ${ }^{76} \mathrm{Kr}$ (middle part) and ${ }^{240} \mathrm{Pu}$ (lower part) at $L=0$. The deformation of the DNS nuclei is taken into account. 


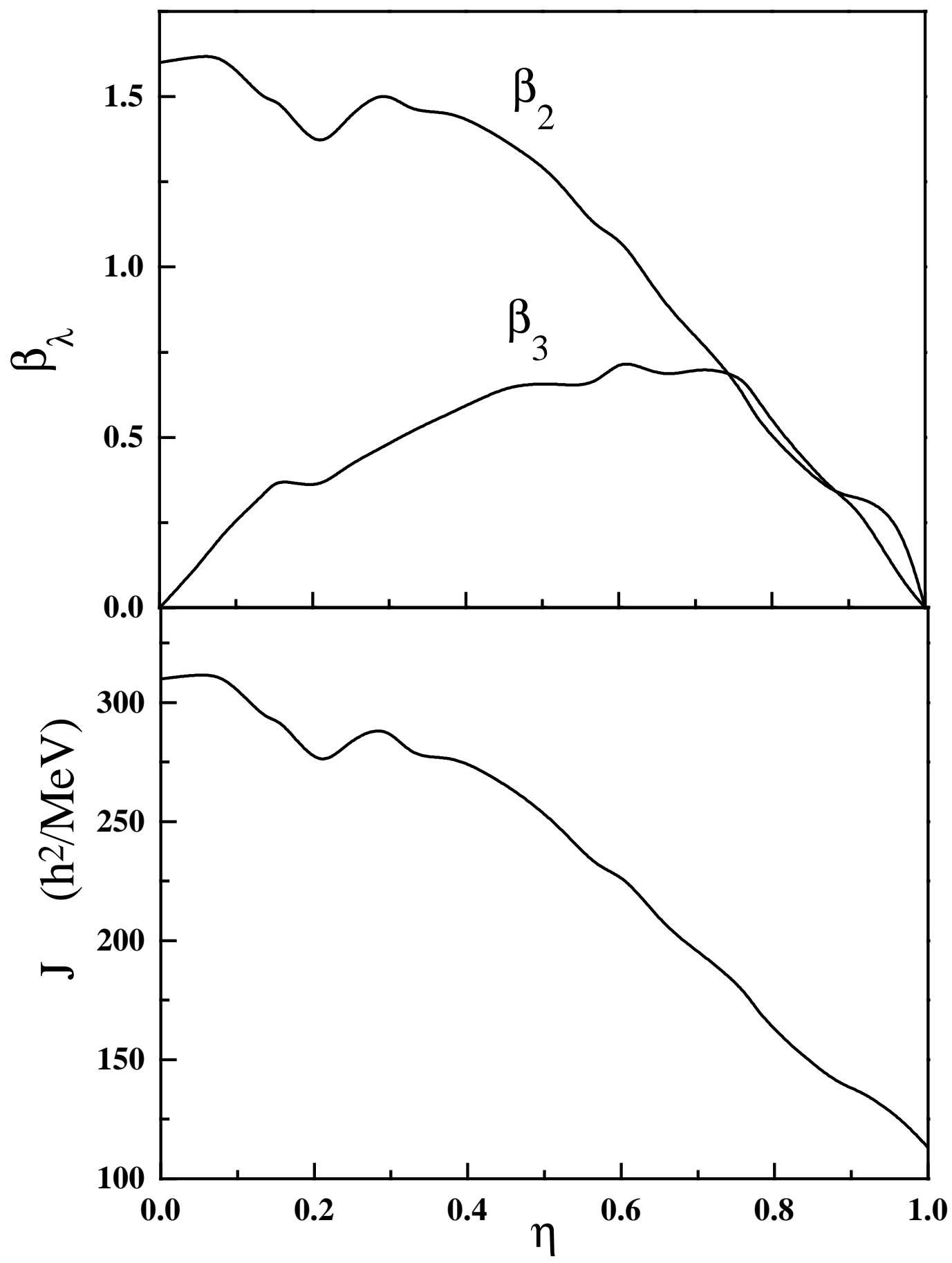

FIG. 6. Deformation parameters $\beta_{2}$ and $\beta_{3}$ (upper part) and moment of inertia $\Im=\Im^{r}$ (lower part) as a function of mass asymmetry $\eta$ of the DNS corresponding to the ${ }^{232}$ Th compound nucleus. 


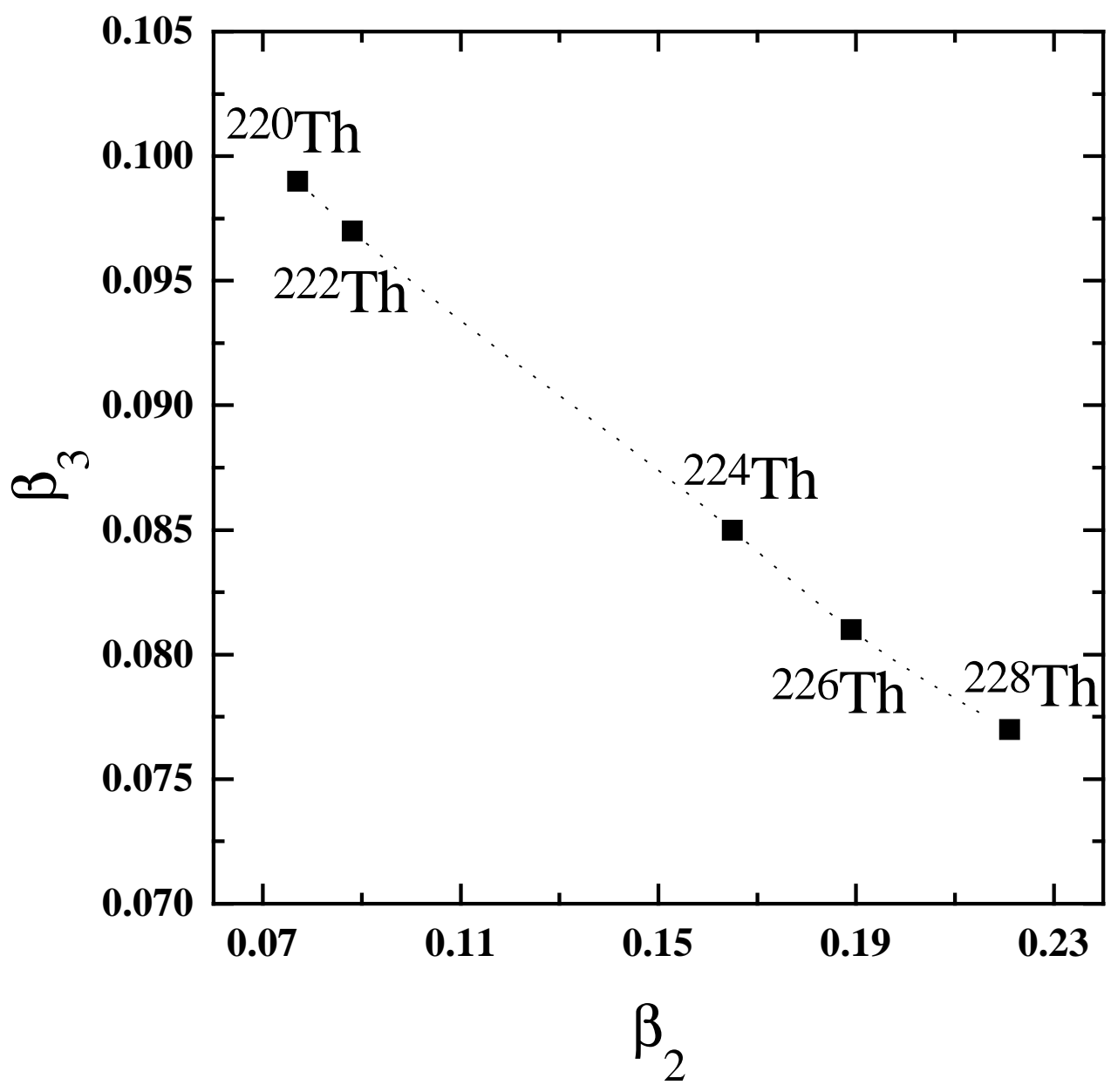

FIG. 7. The $\beta_{2}$ vs. $\beta_{3}$ plot for different isotopes of ${ }^{A}$ Th in the cluster state ${ }^{(A-4)} \mathrm{Ra}+{ }^{4} \mathrm{He}$. 